\title{
A Fiber-Optic Current Sensor for Lightning Measurement Applications
}

\author{
Truong X. Nguyen, Jay J. Ely, George N. Szatkowski \\ NASA Langley Research Center, 1 S. Wright Street, Hampton, VA, USA 23681-0001
}

\begin{abstract}
An optical-fiber sensor based on Faraday Effect is developed for measuring total lightning electric current. It has many unique capabilities not possible with traditional current sensors. Designed for aircraft installation, the sensor is lightweight, non-conducting, structure-conforming, and is immune to electromagnetic interference, hysteresis and saturation. It can also be used on windmills, lightning towers, and can help validate lightning detection network measurements.

Faraday Effect causes light polarization to rotate when the fiber is exposed to a magnetic field in the direction of light propagation. Thus, the magnetic field strength can be determined from the light polarization change. By forming closed fiber loops and applying Ampere's law, measuring the total light rotation yields the total current enclosed. The broadband, dual-detector, reflective polarimetric scheme allows measurement of both DC component and AC waveforms with about $60 \mathrm{~dB}$ dynamic range.

Three sensor systems were built with different sensitivities from different laser wavelengths. Operating at 850nm, the first system uses twisted single-mode fiber and has a $150 \mathrm{~A}-150 \mathrm{KA}$ range. The second system operates at $1550 \mathrm{~nm}$, uses spun polarization maintaining fiber, and can measure $400 \mathrm{~A}-400 \mathrm{KA}$. Both systems were validated with rocket-triggered lightning measurements and achieved excellent results when compared to a resistive shunt. The third system operates at $1310 \mathrm{~nm}$, uses spun polarization maintaining fiber, and can measure approximately $300 \mathrm{~A}-300 \mathrm{KA}$. High current measurements up to $200 \mathrm{KA}$ were demonstrated at a commercial lightning test facility. The system was recently installed on an aircraft and flown near icing weather conditions.
\end{abstract}

Keywords: Faraday Effect, current sensor, fiber optic, lightning

\section{INTRODUCTION}

Characterization of in-flight lightning return stroke current could provide useful data in developing aircraft protection methods and test parameters. In addition, directly measuring lightning current allows for real-time on-aircraft lightning detection and damage assessment. There is a need for a sensor that can measure total current directly and is easily installable on aircraft. These capabilities are not possible with traditional sensors. Past flight efforts [1-3] to characterize lightning parameters used sensors that required extensive aircraft modifications not practical for standard commercial aircraft (i.e. current shunt or I-dot sensor). Many sensors could only measure local parameters such as surface current, electric field or magnetic field, and extensive computational modeling and analysis were required to approximate lightning current [2,3]. In addition, they could not measure low frequency (near DC) components associate with continuing current and heating damage. Traditional sensors that are capable of measuring large current in lightning are often not practical for aircraft installation due to aircraft's safety, weight and aerodynamic performance requirements.

The fiber-optic current sensor is based on Faraday (Rotation) Effect and can overcome most limitations of traditional current sensors, making accurate direct aircraft lightning measurement feasible and affordable. This type of sensor has been under development by the power industry for decades. Many products are starting to be commercially available that meet power industry's stringent accuracy standards. Faraday Effect causes light's polarization plane in a medium to rotate when the medium is exposed to a magnetic field in the direction of light propagation. Using optical fiber as the propagation medium and by forming closed fiber loops, measuring the total light rotation would result in the total current enclosed. Thus, total current is measured by installing one or more fiber loops around the structure of interest. It is noted that the sensing element is an optical fiber, thus termed "intrinsic" sensor. In contrast, in "extrinsic" sensor optical fiber is only used for relaying signal from a remote sensor.

Unlike the well-known Rogowski coil, the optical-fiber sensor is self-integrating - thus no integrator is needed, and the DC component can be measured with suitable electronics. It does not suffer from self-resonance, hysteresis and saturation 
as with Rogowski coils or current transformers. It is not susceptible to arcing/sparking from high voltage and current. Measurement may be performed at arbitrary locations along the structure. This permits measurement of swept-stroke current that is not possible for sensors installed at aircraft's nose or wing-tip locations.

Compared to traditional sensors, there are many unique characteristics making it ideal for aircraft installation. It is small, light-weight and flexible, yet can accurately measure large lightning current. It is highly conformable and can fit tightly onto structure's surface contours. Installation is simple and non-intrusive, simply by wrapping the thin fiber one or more times around the structure to be measured. Being non-conductive, the sensing fiber can be safely routed directly into the aircraft fuselage or through flammable regions. The sensor use is highly versatile, as the same fiber can be used on both small and large structures, given sufficient length and bandwidth. Measurement sensitivity may be tailored by varying the number of fiber-turns around the conductor.

There are limitations, however. Fiber choice is limited, as most commonly available fibers are based on silica in which the Faraday Effect is weak. However, the weak effect makes the sensor highly suitable for large current such as in lightning. There are slight sensitivities to bend/vibration and temperature variations, but there are compensation methods. Glass fiber can be fragile and needs suitable protection.

Aircraft installations with their strict safety and aerodynamic performance requirements would benefit the most from this new sensor. The flight system would be greatly simplified by the direct total current measurement and the installation flexibility. No longer required are multiple external local field sensors and optical converters (for safety). Avoided are extensive computer modeling and analysis solving inverse problems pertaining to one specific aircraft. Fig. 1 illustrates fiber-optic sensor loops installation in contrast with traditional B-Dot sensors that can only measure local magnetic fields. Results in this paper show excellent accuracy comparable to many reference sensors. The sensor's many advantages could also benefit other direct lightning measurements on small buildings, windmills, or lightning towers.

This fiber-optic current sensor benefits greatly from earlier development work by the industry. The approach chosen in this effort is suited for lightning parameters and is a compromises between bandwidth, measurement range and accuracy. In addition, the implementation approach gives emphasis to simplicity, low-cost, and the ability to measure (near) DC component in continuing current. Prototypes presented in this paper were developed without specialized optical tools.

Described in the paper are the basic sensor design, implementation, laboratory testing, and field testing with rockettriggered lightning. Three similar prototype systems discussed are based on $850 \mathrm{~nm}, 1310 \mathrm{~nm}$ and $1550 \mathrm{~nm}$ laser wavelengths. The measurement ranges achieved are approximately $150 \mathrm{~A}-150 \mathrm{KA}, 300 \mathrm{~A}-300 \mathrm{kA}$ and $400 \mathrm{~A}-400 \mathrm{kA}$, respectively, a roughly $60 \mathrm{~dB}$ range. Due to damage risks to aircraft from lightning strikes, none of the systems has been flown into thunderstorms to date. However, one system was installed on an aircraft and flown near thunderstorms on three flights. The purpose was to demonstrate the simple sensor installation and to assess the system's immunity to false triggers. Additional details are discussed later in the paper.

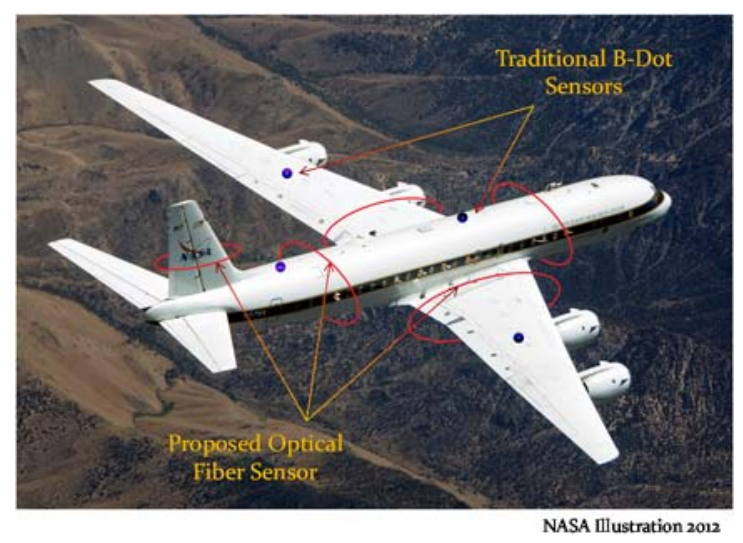

Figure 1. Optical-fiber sensors aircraft installation. 


\section{FIBER-OPTIC CURRENT SENSOR}

\subsection{Basic Sensor Operation}

The Faraday Effect causes linearly polarized light in the sensing fiber to rotate when exposed to an external magnetic field in the direction of light propagation [4-8]. The effect in an optical fiber is illustrated in Fig. 2. The total polarization plane rotation $\phi$ is

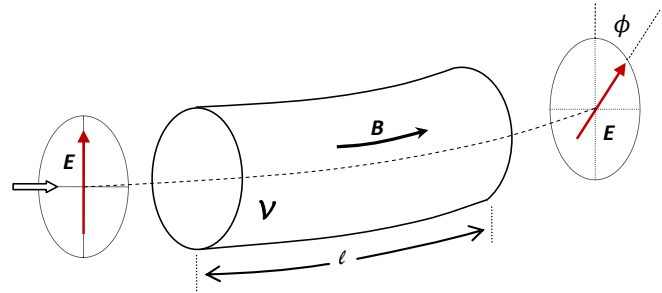

Figure 2: Faraday Effect in optical fiber.

$$
\phi=V \int B \cdot d l=\mu_{0} \int H \cdot d l
$$

where $\mu_{0}$ is the free-space permeability; $V$ is the Verdet constant in radians/(meter-Tesla); $\mu_{0} V$ is the combined permeability Verdet constant (radians/ampere); $\boldsymbol{B}$ is magnetic flux density in Tesla (T); $\boldsymbol{H}$ is the magnetic field (amperes/meter); and $\boldsymbol{I}$ (in meters) is the light and magnetic field interaction path length. Forming $N$ fiber loops and applying Ampere's law yields result in $\phi$ being directly proportional to the total current enclosed $I$ (Eq. 2). Thus, $I$ can be determined by measuring $\phi$. As shown in Eq. 2, the sensor is self-integrating, no additonal integration is needed.

$$
\begin{aligned}
\phi & =\mu_{0} V \oint H \cdot d l, \\
& =\mu_{0} V N I .
\end{aligned}
$$

\subsection{Polarimetric Detection Scheme}

The scheme to measure polarization change induced by current is illustrated in Fig. 3. Linearly polarized light is launched from a super-luminescence diode (SLD) onto the sensing fiber at 4 that forms closed loops around a structure of interest. As light reflects from the Faraday Mirror at 6, its polarization is rotated 90 degrees relative to the incident light. This Faraday mirror implementation makes the system less sensitive to bending, since stress induced effects on polarization in one direction are canceled by the same effects on the orthogonal polarization in the reverse direction [5]. As reflected light traces back to the non-polarizing beam splitter (NBS) at 3, half of the power is reflected toward the half-wave plate (HWP) at 7 and the polarizing beam splitter (PBS) at 8. Alternately, the PBS can be implemented in a fiber assembly. Exiting the PBS, light power in the two orthogonal polarizations are measured by two photo-detectors D1 and D2 at 9. The difference in optical powers at 9 is measured with a balanced detector, whose output voltage being proportional the difference between the two detectors' outputs. The HWP at 7 allows one to rotate and align the beam polarization incident on the PBS.

Ideally, at zero current the incident polarization should be at $45^{\circ}$ relative to the PBS's two orthogonal principle polarization axes, so that beam power is divided equally between the two optical detectors. With no current the balanced detector's output should be at zero volts. With current, Faraday Effect causes the light's polarization to rotate, causing power imbalance at the two detectors, leading to a voltage output at the balanced detector.

This setup is referred to as a reflective scheme since a mirror is incorporated and light travels round-trip. With the Faraday Effect being non-reciprocal, the Faraday rotation is doubled relative to single-pass scheme. The responses at the two detectors should ideally be $(\mathrm{a}),(\mathrm{b})=0.5\left[1 \pm \sin \left(4 \mu_{0} \mathrm{VNI}\right)\right]$ for a reflective scheme. Mathematic operation difference-oversum, $(\mathrm{c})=(\mathrm{a}-\mathrm{b}) /(\mathrm{a}+\mathrm{b})$, yields

$$
\begin{aligned}
& (\mathrm{c})=\sin \left(4 \mu_{0} V N I\right), \text { or } \\
& N I=\sin ^{-1}(\mathrm{c}) /\left(4 \mu_{0} V\right),
\end{aligned}
$$


where equivalent current $N I$ (in unit Ampere-turn) is defined as number of loops $N$ times the current $\mathrm{I}$, and $\mu_{0} V=2.5$ $\mu \mathrm{rad} / \mathrm{A}$ at $850 \mathrm{~nm}, 1.01 \mu \mathrm{rad} / \mathrm{A}$ at $1310 \mathrm{~nm}$ and $0.718 \mu \mathrm{rad} / \mathrm{A}$ at $1550 \mathrm{~nm}$ [4] for the systems described in this paper.

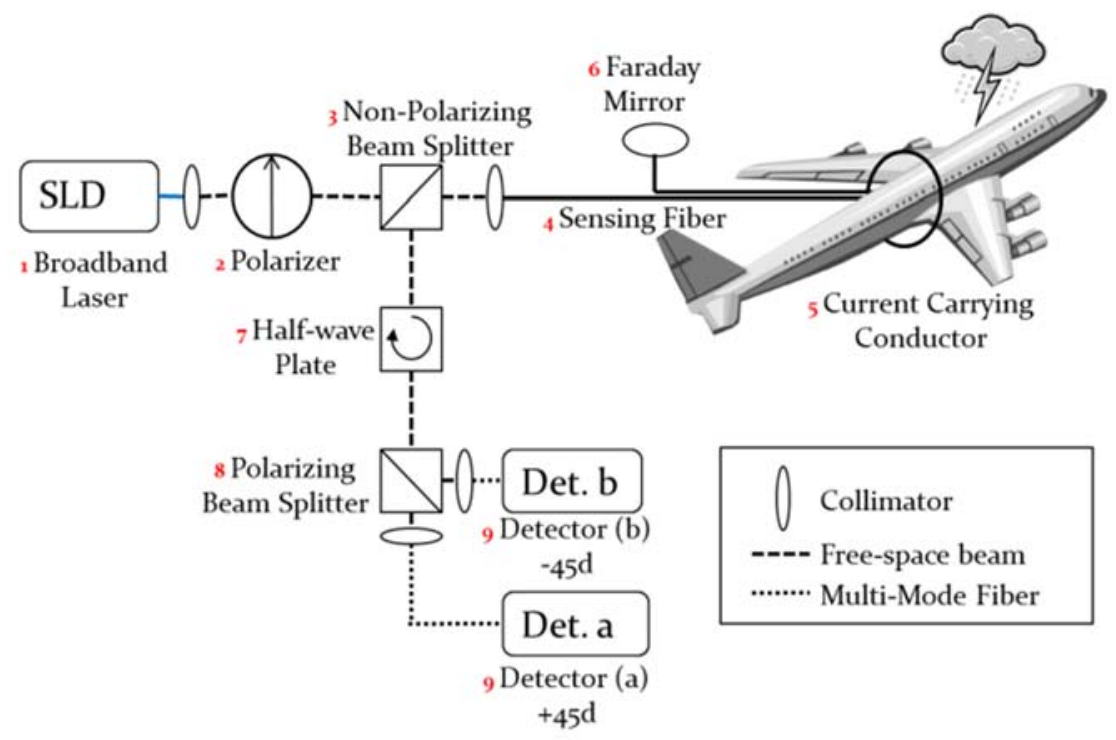

Figure 3. Optical detection scheme.

Fig. 4 illustrates the ideal responses at 1310nm, with the response curves (a) and (b) being voltage outputs from the two optical detectors. The difference-over-sum operation, $(c)=(a-b) /(a+b)$, would yield a response that is more sensitive (higher slope) than either response curve (a) or (b), with zero crossing at zero current, and has larger dynamic range due to common-mode noise subtraction. Current I or equivalent current NI is then computed from (c) using eq. (4).

The typical operating range is where the curve (c) increases monotonically, or about $-350 \mathrm{kA}$ to $+350 \mathrm{kA}$. In this range the response and current correspond one-to-one. In the systems described, the non-ideal fiber medium and optical components distort the curves. The practical range is slightly reduced to about $-300 \mathrm{kA}$ to $+300 \mathrm{kA}$, as shown in the next section.

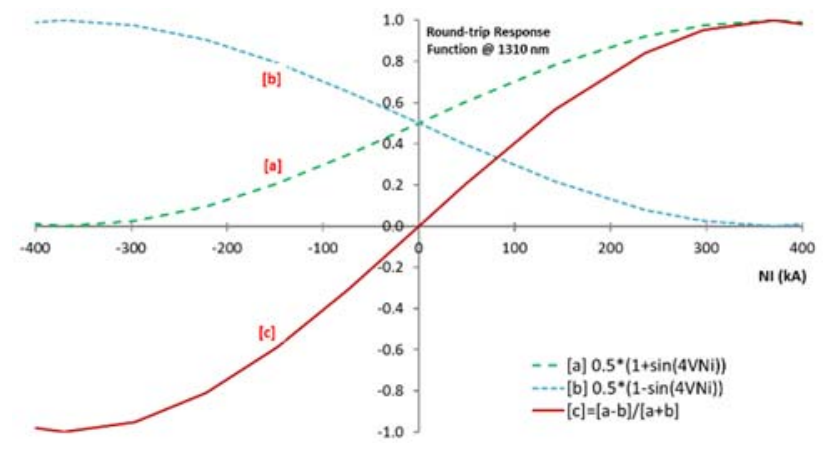

Figure 4. Ideal sensor responses at $1310 \mathrm{~nm}$.

Discussions for the $850 \mathrm{~nm}$ and $1550 \mathrm{~nm}$ systems are similar, only with the current values scaled appropriately for different $\mu_{0} \mathrm{~V}$ value of each system. The $1550 \mathrm{~nm}$-based system is slightly less sensitive due to the lower Verdet constant at this wavelength, so it can measure slightly larger current. The practical range is approximately $+/-400 \mathrm{kA}$. The $850 \mathrm{~nm}$ system is slightly more sensitive, with the practical range approximately $+/-150 \mathrm{kA}$. The design, construction and characteristics otherwise are similar to the $1310 \mathrm{~nm}$ system. 
Over-current will not damage the sensor as light polarization will simply rotate beyond the intended range. The solution to the $\sin ^{-1}$ function in (4) would be ambiguous. However, there are variations of the detection scheme that permit measurement of very large current [6]. These practices are not necessary since for most direct lightning are within the 150 $\mathrm{kA}, 300 \mathrm{kA}$ and $400 \mathrm{kA}$ ranges for systems described. One simply choses the appropriate wavelength for the desired current range.

It is important that light's state-of-polarization (linear) is maintained in the fiber during light transit. This is achieved with fiber selection. The $1310 \mathrm{~nm}$ and $1550 \mathrm{~nm}$ systems use two different commercial highly-spun polarization-maintaining (PM) fibers [7], which are the result of twisting PM fibers during manufacturing. Fiber twisting helps hold the state-ofpolarization that otherwise would be destroyed in a typical fiber. The twist rate is about $4-5 \mathrm{~mm}$ per turn. Additional information on fiber-optic current sensor can be found in [4-8]. In this implementation, linearly polarized light is launched onto the spun PM fiber at approximately 60 degrees relative to the fiber's slow axes. The $850 \mathrm{~nm}$ system uses standard single-mode telecom fiber that is twisted at 20 twists/meter. The twist is held in place at both ends inside $3 \mathrm{~mm}$ diameter jacket using epoxy, similar to that used in [6].

\subsection{Calibration and Data Correction}

Calibration is performed in laboratory by relating the sensor's response curve to data from reference sensors, and a calibration function is developed. The reference sensors used include a Rogowski coil (with an electronic integrator) and a ferrite-based Pearson ${ }^{\mathrm{TM}}$ current transformer (CT). Fig. 5 compares the $1310 \mathrm{~nm}$ system against two reference sensors. Peak equivalent current from the Faraday sensor is displayed on the vertical axes against reference sensors on the horizontal axes. Large equivalent currents in the figure were achieved by using multiple fiber turns and/or a wire coil - details are described in the next section.

Ideally the Faraday sensor data would fall on the straight diagonal line labeled "ideal" representing (1:1) correspondence with the two reference sensors. Instead, the data follow the curve labeled "uncorrected". This non-ideal response is due to the reduced sensitivity in the spun fiber [7], relative to ideal medium of the same material - along with some light depolarization from the non-ideal fiber medium and optical components. Thus, it is important that the sensor is calibrated to develop a correction factor or function.

A correction function was developed by spline-fitting the "uncorrected" curve in Fig. $5\left(9^{\text {th }}\right.$ order polynomial function was used). The purpose was to map the Faraday sensor response curve to the "ideal" curve. Fig. 5 shows the "corrected" response curve aligns well with the "ideal" diagonal line. That same function is then applied to subsequent measurements to achieve corrected results. Data interpolation may also be used instead of curve fitting. However, neither approach is perfect as some small error may remain. In addition, only a simple correction factor may be needed if measurement is restricted to the linear region, i.e. $100 \mathrm{kA}$ or less for the $1310 \mathrm{~nm}$ system. The same discussion is extended to include the $850 \mathrm{~nm}$ and $1550 \mathrm{~nm}$ systems.

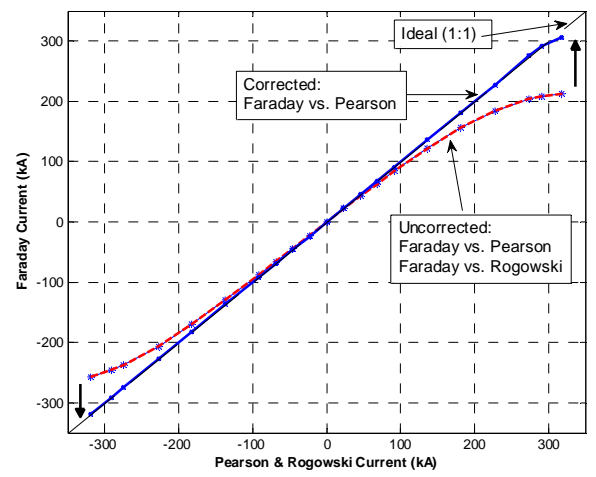

Figure 5. Example of corrected and un-corrected response curves for 1310nm system

\subsection{Sensor Bandwidth}

Bandwidth of a sensor system is limited by the lowest bandwidth of its components. For the fiber sensor component, it is limited by the light transit time in the interaction length of the fiber. This bandwidth limitation is to ensure the total transit time is much faster than the signal change rate. The fiber interaction length in the bandwidth consideration includes the roundtrip length around the conductor and includes the length to and from the Faraday sensor. The 3-dB sensor bandwidth 
BW is [4] (generalized to interaction length rather than diameter): $B W \approx \frac{0.44}{t} \approx 0.44 c / n L$, where $t$ is transit time, $c$ is the speed of light in free space, $n$ is the index of refraction in fiber material $(n=1.5)$, and $L$ is the interaction length (double of fiber length for reflective scheme).

Table 1 computes the maximum fiber length and structure dimensions for different bandwidths. Aircraft thin structures may include wings and tail surfaces, while round structures may include fuselage, engine, etc. For reference, fuselage outside diameters for various aircraft (by averaging the width and height) include: Airbus A380: $7.8 \mathrm{~m}$; Boeing 767: 5.3 $\mathrm{m}$; Boeing 737:3.8 m. Since most of lightning energy is contained in spectrum region far below 1-2 MHz, the table shows there is sufficient sensor bandwidth even for the fuselage of the largest passenger aircraft, the Airbus A380.

Table 1. Structure Dimensions vs. Sensor Bandwidths.

\begin{tabular}{|c|c|c|c|}
\hline $\begin{array}{c}\text { 3-dB } \\
\text { Bandwidth } \\
\text { (MHz) }\end{array}$ & $\begin{array}{c}\text { Max. Fiber } \\
\text { Length (m) }\end{array}$ & $\begin{array}{c}\text { Max. Thin } \\
\text { Structure } \\
\text { Dimension (m) }\end{array}$ & $\begin{array}{c}\text { Max. Round } \\
\text { Structure } \\
\text { Diameter (m) }\end{array}$ \\
\hline 1 & 44 & 22 & 14 \\
\hline 2 & 22 & 11 & 7 \\
\hline 4 & 11 & 5.5 & 3.5 \\
\hline 10 & 4.4 & 2.2 & 1.4 \\
\hline 20 & 2.2 & 1.1 & 0.7 \\
\hline
\end{tabular}

\section{LABORATORY DEMONSTRATIONS}

This section describes various laboratory lightning measurement demonstrations. These include 1) calibration and data correction with simulated large current, 2) direct current measurement of large current up to $200 \mathrm{kA}$ performed at a commercial laboratory, 3) current measurement on a simulated aircraft fuselage, and 4) low-level indirect lighting current inside a simulated fuselage. Demonstrations measuring rocket-triggered-lightning are described in the next section.

\subsection{Calibration and Data Correction}

For the purpose of calibrating and testing the sensors, it is difficult and unsafe to achieve in laboratory current levels up to maximum ranges, namely $150 \mathrm{kA}, 300 \mathrm{kA}$ and $400 \mathrm{kA}$ for the three sensor systems. One acceptable approach to producing the associated optical effects in the fiber is by using multiple fiber loops and/or a multi-turn conductor coil. Multiple fiber loops and/or multi-turn coil amplify the Faraday rotation beyond the amount produced by a single fiber loop around a single conductor. The amplification factor is the multiplication of the number of fiber-loops and the number of wire turns used. Fig. 6 illustrates a typical setup. Laboratory tests show using multiple fiber loops, a multi-turn coil, or combinations of both yield the same response curves. Using a wire coil having a high number of turns may distort the injected waveform due to the increased inductance. In contrast, a high number of fiber loops does not affect the current waveform but a longer sensing fiber is needed. For simplicity, $N$ is redefined from Eq. (4) to be the product of the number of fiber loops and the number of wire turns. The product $N I$ is referred to as equivalent current as previously stated in Eq. (4).

Fig. 6 shows results comparison between the $1310 \mathrm{~nm}$ system and the reference sensors. The equivalent currents $N I$ are about $5 \mathrm{kA} \bullet$ turns and $300 \mathrm{kA} \bullet$ turns. The same calibration correction function was used in both cases and good results were achieved. Since the reference Rogowski coil and Pearson current transformer (CT) only measured current on one wireturn, their results were numerically scaled up by the factor $N$ for the comparison. Similarly good results were achieve for the $850 \mathrm{~nm}$ and $1550 \mathrm{~nm}$ systems. 

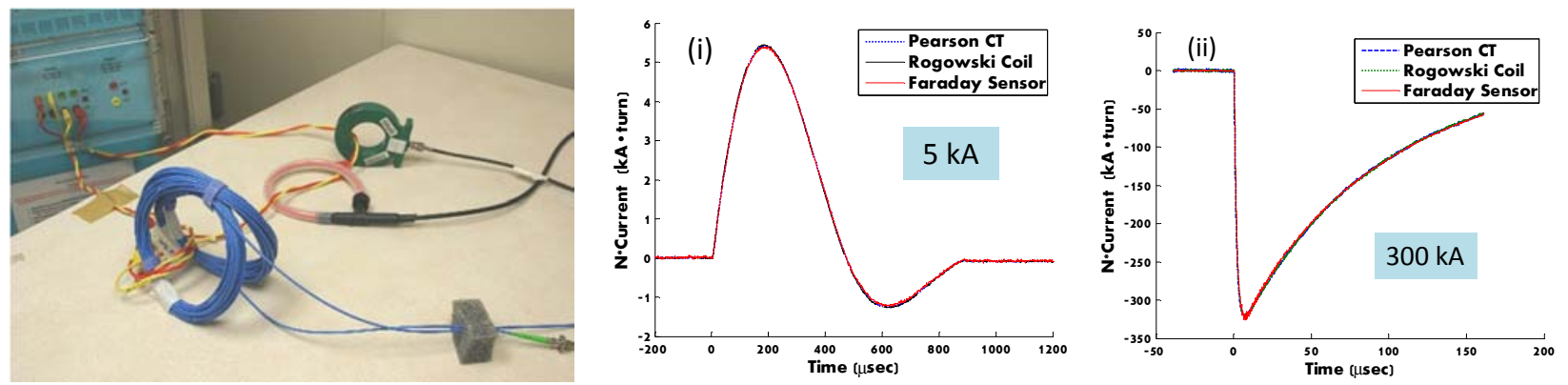

Figure 6. Using multi-turn coil and multiple fiber loops to achieve high current effects (5 kA and $300 \mathrm{kA})$. (i) 49-turn coil and one fiber loop $(N=49)$, and (ii) 3-turn coil and 28 fiber loops $(N=3 * 28=84)$.

\subsection{Direct Lightning Large Current Measurement}

The $1310 \mathrm{~nm}$ sensor system was evaluated for large current performance using only one fiber loop around one conductor $(N=1)$. Using one fiber loop would be similar to installation external to an aircraft fuselage, a large structure, or a lightning tower. The tests were performed at a commercial lightning test facility using standard aircraft lightning test waveforms and include components D, B and C [9]. Test current amplitudes were 20, 40,100 kA with double-exponential waveforms, and $200 \mathrm{kA}$ with damped sinusoidal waveform. The damped sinusoidal waveform was necessary due to the test facility's limited abilities to generate high current unipolar waveforms. The measurement piggybacked on a separate effort to evaluate lightning effects on composite panels.

The setup shown in Fig. 7 included the fiber forming one closed loop around a flat metal plate (about $0.5 \mathrm{~m}$ wide) carrying the return current that exits the composite panels under test. The Faraday sensor results were compared against the sum of four Pearson CTs measuring currents exiting the four sides of the composite panel. In a good setup, closing the fiber loop at the optical box would provide good isolation to reject stray magnetic fields. However, this was not possible due cable routing restrictions. A section of the fiber was routed near the lightning test zone on its way to the optical box; thus, some error was anticipated.

Comparison results for $100 \mathrm{kA}$ and $200 \mathrm{kA}$ peak currents are shown in Fig. 7 and are reasonably good considering the non-optimal setup. The errors are about $3-10 \%$ depending on the routing of the exposed fiber section through the lightning test zone. It is clear that the Faraday sensor is capable of directly measuring $200 \mathrm{kA}$ current using just one loop, and that a better setup could lead to even better comparisons. The $20 \mathrm{kA}$ and $40 \mathrm{kA}$ comparisons are similar to the $100 \mathrm{kA}$ results.
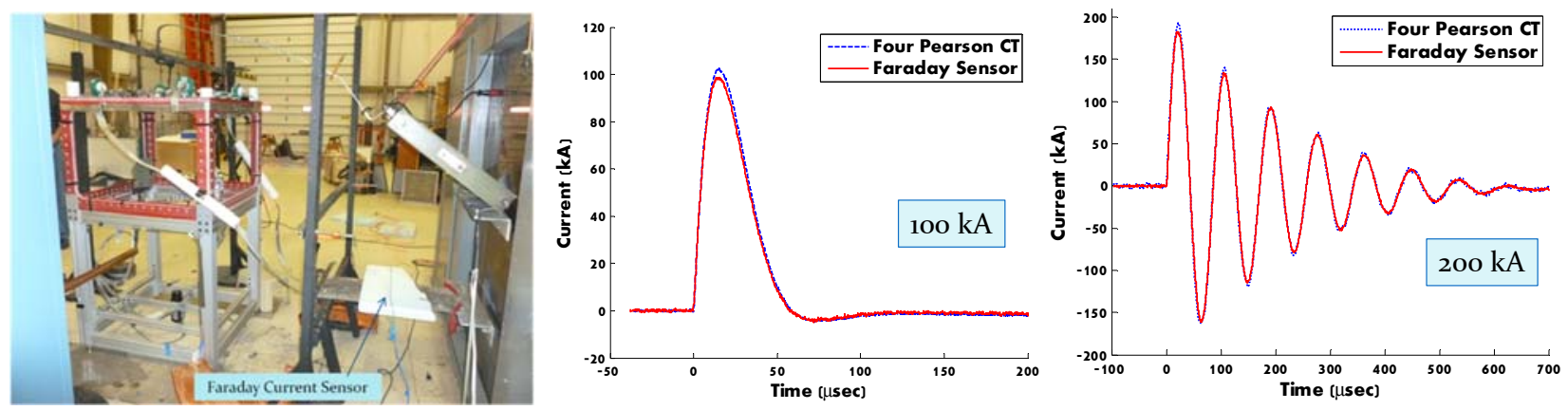

Figure 7. A high-current measurement setup and data for $100 \mathrm{kA}$ and $200 \mathrm{kA}$ on a current return plate.

\subsection{Measurement on a Simulated Fuselage}

Fig. 8 illustrates a setup measuring current on a $1.2 \mathrm{~m}$ diameter aluminum cylinder that simulates an aircraft fuselage or round lightning tower. Current lightning waveforms from $250 \mathrm{~A}$ to $4 \mathrm{kA}$ (amplitudes limited by laboratory equipment) were injected onto the cylinder at the bottom left location. Return currents exits from the cylinder at bottom right.

The $15 \mathrm{~m}$ sensing fiber form a single loop around the cylinder, with both ends co-routed to the optical box located $4 \mathrm{~m}$ away in the foreground. As shown in the figure, the fiber closed the loop at the optical box without any unpaired fiber section, 
thus good isolation was achieved. A Pearson CT and a Rogowski coil provide reference comparison data. Fig. 8 shows good results for both the $250 \mathrm{~A}$ and $4 \mathrm{kA}$ tests.

Noise is observable in the 250 A measurement, illustrating the low level sensitivity limit. The dominant source of noise is the SLD, which is a wideband optical noise source. Noise reduction techniques implemented include using the balanced detector for common mode noise subtraction and a $1.9 \mathrm{MHz}$ low-pass filter. In addition, a small moving-window data averaging is implemented in post-processing, i.e. 11-point window out of 10,000-points data length. A $60 \mathrm{~dB}$ range could be achieved with this setup.
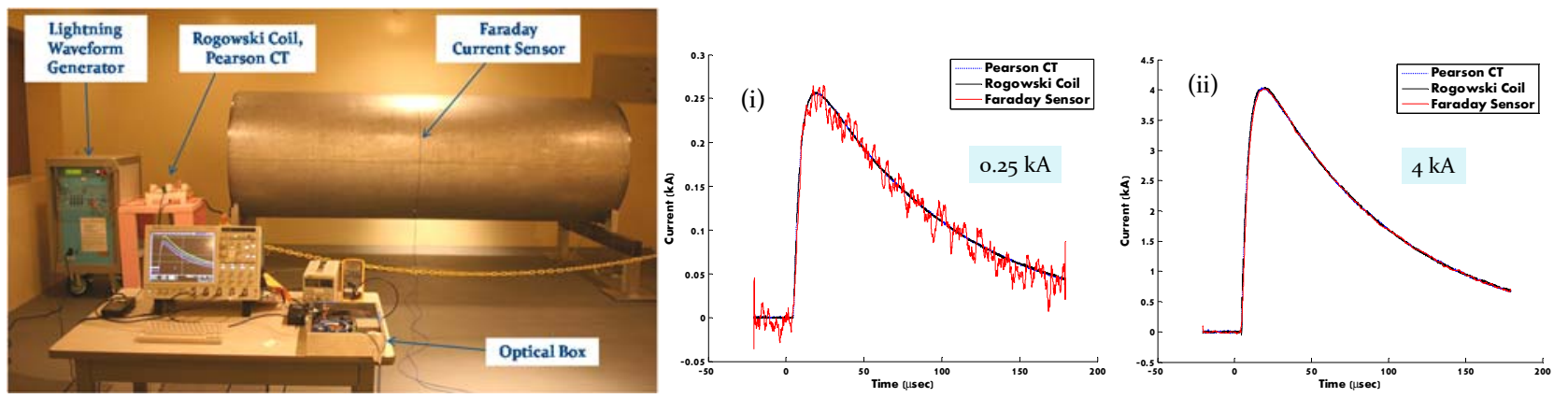

Figure 8. $250 \mathrm{~A}$ and $4 \mathrm{kA}$ peak current measurements on a $1.2 \mathrm{~m}$ (4 feet) diameter structure.

\subsection{Indirect Lightning Internal Current Measurement}

Fig. 9 demonstrates excellent isolation for low current measurement on an internal structure. This setup simulates induced current on equipment or wire bundles in the presence of significantly larger current flowing on the outer aluminum structure. About $2 \mathrm{kA}$ peak total current was injected on the aluminum cylinder, with about $140 \mathrm{~A}$ flows on the internal box. Generally good comparison can be observed even though the Faraday sensor's data were close to the noise floor.

Excellent results were also achieved when the fiber loop was expanded all the way to the metal frame of the aluminum structure. This illustrates the ability to measure the composite sum current on all internal cables and structures enclosed by the fiber loop. This ability could help determine the occurrence and amplitude of lightning induced current, and whether maintenance service may be needed after a strike.
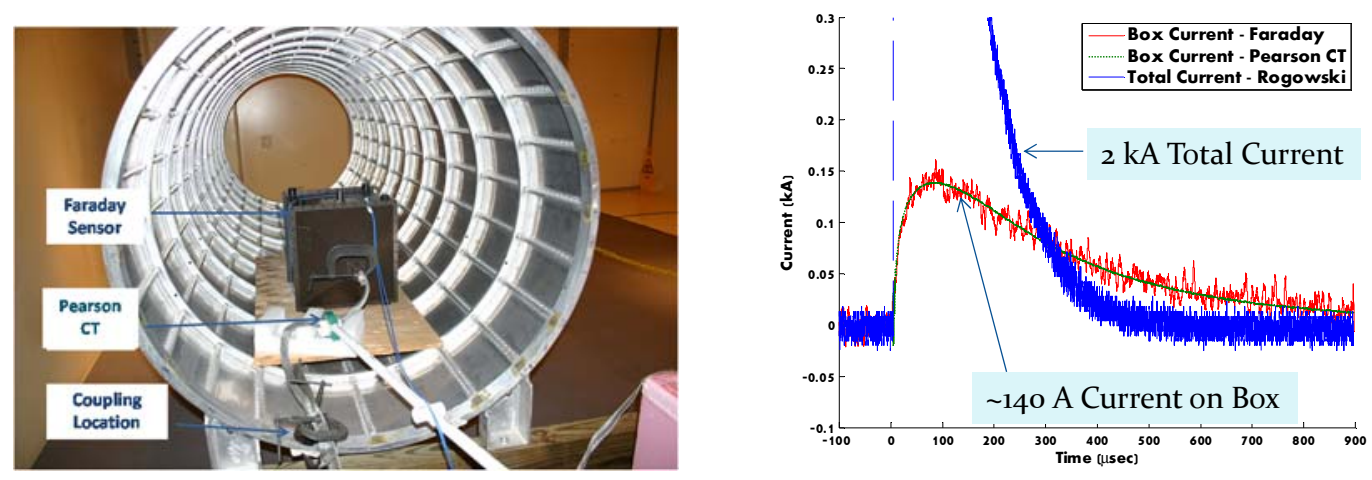

Figure 9. Low-level measurement on an internal structure in presence of larger current on the external structure.

\section{ROCKET-TRIGGERED LIGHTNING MEASUREMENT}

The $850 \mathrm{~nm}$ and $1550 \mathrm{~nm}$ systems were demonstrated measuring rocket-triggered lightning in 2011 and 2012, respectively. The measurements were performed at the International Center for Lightning Research and Testing (ICLRT) facility at Camp Blanding, Florida, U.S.A. [10]. Both systems yielded good comparison results. However, being in the weather the $850 \mathrm{~nm}$ system show symptoms of corrosion in the cabling that affected amplitude readings, though the waveform comparisons remained excellent. The discussion below is restricted to the $1550 \mathrm{~nm}$ due to space limitation. The setup for the $850 \mathrm{~nm}$ system was similar.

In the setup shown in Fig. 10, triggered-lightning flashes would attach to the intercepting ring, and lightning current would travel to the ground via a down-conductor. A resistive shunt (T\&M Model R-7000-10) in-line with the down conductor 
provided reference measurements. The sensing fiber formed four loops around the same down-conductor. The remaining fiber segments at the two ends were co-routed radially away from the site. One end was connected to the supporting optical box $12 \mathrm{~m}$ away. At the other end the Faraday mirror was buried in the ground $4 \mathrm{~m}$ away from the fiber loops to minimize temperature variations on the mirror. Data were recorded using a commercial 14-bit digitizer sampling at $100 \mathrm{MHz}$. The sensors and digitizers were powered by batteries.

Due to insufficient fiber length $(25 \mathrm{~m})$, the Faraday mirror was not positioned near the optical box. About $8 \mathrm{~m}$ (from the Faraday mirror to the optical box) section of the sensing fiber was "unpaired", potentially exposed to fields from lightning flashes and ground currents that would normally be canceled with a closed loop. However, by routing the fiber in the radial direction away from the site, magnetic field components in the direction of the fiber are expected to be minimized, reducing any undesirable effects. The fiber was also protected from wild animals or being trampled on using combinations of rain gutters and plastic braided sleeves.

The system successfully measured rocket-triggered lightning with excellent result comparisons. With the four fiber loops $(\mathrm{N}=4)$ used, the measurement range for the sensor was 100A - 100 kA. Fig. 11 illustrates the representative results. Also shown is low-level comparison to highlight the measurement sensitivity and the ability to measure continuing current. Data were slightly smoothed for noise reduction. The results are nearly identical between the two sensors, except for minor peak amplitude differences in some data (up to 500A) attributable to interference from ground lightning current to electrical cabling. The setup for the $850 \mathrm{~nm}$ system was similar with the optical box shown in Fig. 12 along with some measurement results. The good results illustrate the systems can successfully operate in realistic lightning environments. Additional sensor validation data can be found in [11-13].
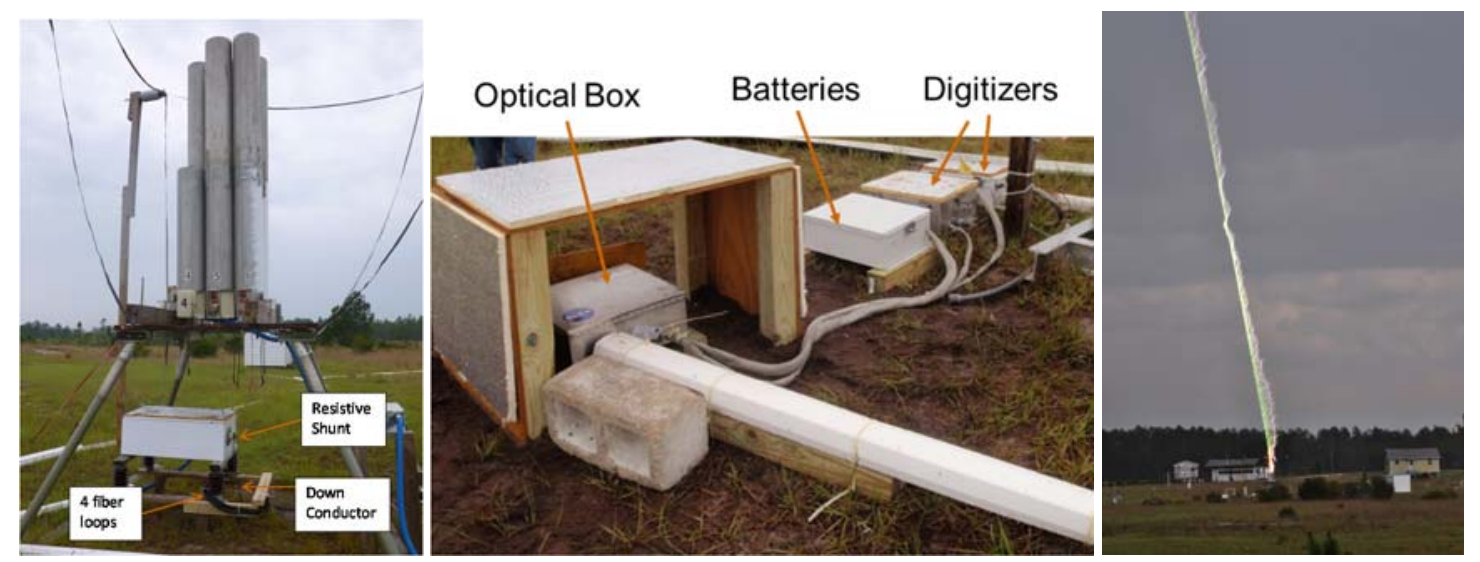

Figure 10. Rocket-triggered lightning current measurement setup at ICLRT with the $1550 \mathrm{~nm}$ system. Triggered Lightning flash (right; with permission from ICLRT 2011)
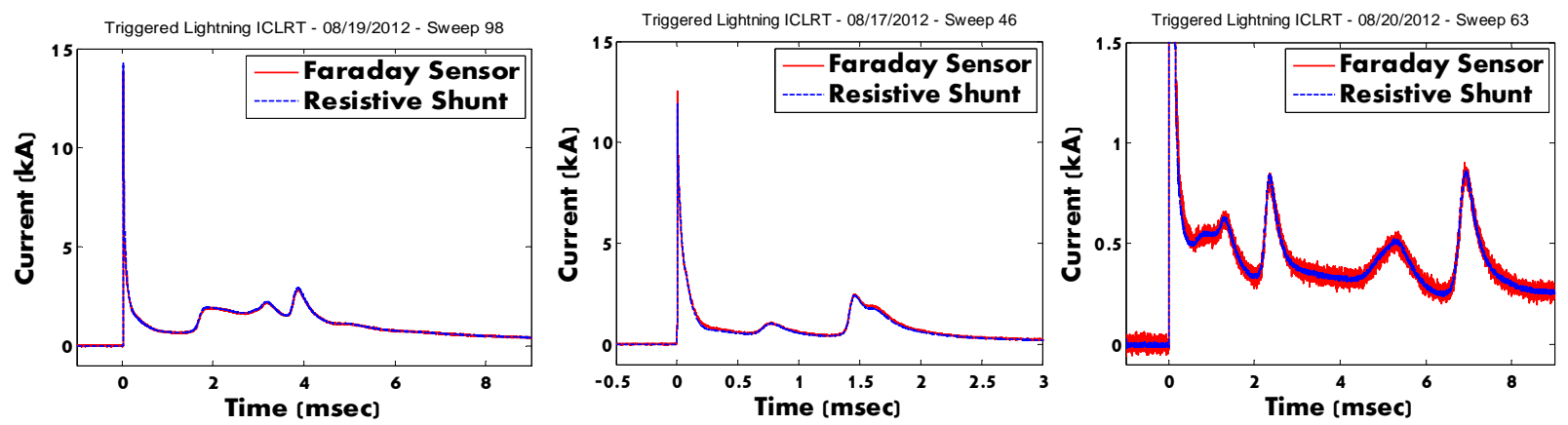

Figure 11. Sample triggered lightning measurements results and low-level comparison using the 1550nm sensor system. 

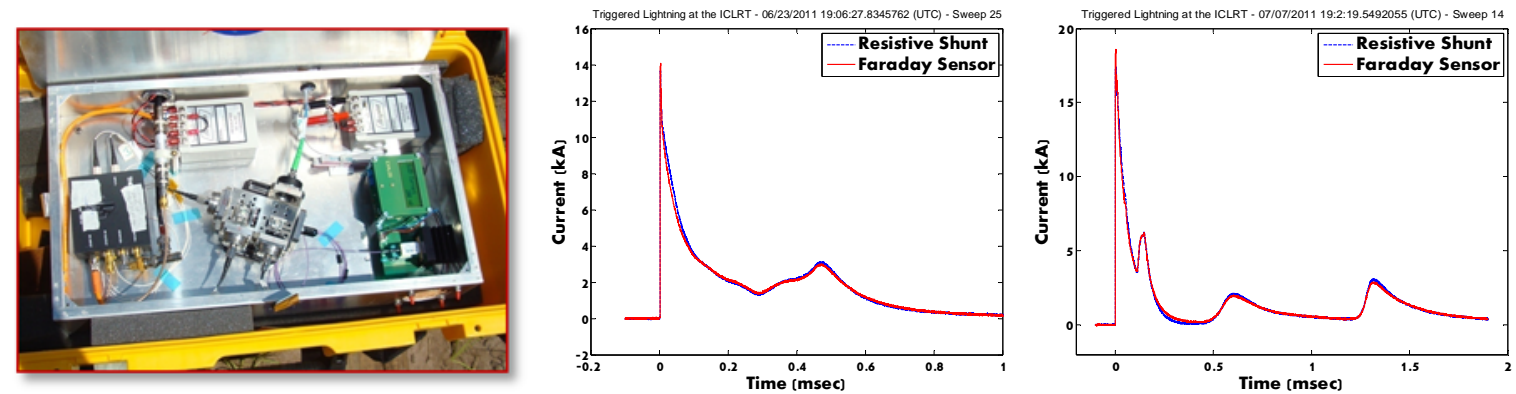

Figure 12. Sample triggered lightning measurements results using the $850 \mathrm{~nm}$ sensor system. Faraday senson data in the far right plot was scaled down to $90 \%$ for good comparison. The amplitude error was caused output cable corosion that lead to higher termination impedance than $50 \mathrm{ohms}$.

\section{AIRCRAFT INSTALLATION DEMONSTRATION}

This section describes a flight demonstration using the $1310 \mathrm{~nm}$ system. The setup piggybacked on another flight experiment with flight paths taking the aircraft close to convective clouds. Though the aircraft was not authorized to fly directly into thunderstorms to be struck by lightning, the goals were to demonstrate ease of sensor installation, to observe the system's behaviors in flight environments, and to determine if the system was susceptible to false triggering as it was the case with field-based sensors in previous flight experiments.

A desire setup includes more than one sensors, each measuring different aircraft structures. This demonstration used only one sensor measuring current on the fuselage in front of the wings section. Fig. 13 illustrates the fiber routing and the final aircraft installation. The $40 \mathrm{~m}$ sensing fiber exited the fuselage through an unused antenna port on top, routed forward and formed one closed loop around the fuselage in front of the wing section. The fiber was then routed toward the back and re-entered the fuselage through the same hole. The hole was protected with a blank plate and a spacer plate with gap for the fiber as shown in Fig. 14.

The fiber was secured to the fuselage with 2"-wide polyurethane protective tape designed for aircraft external installation. The tape was previously tested on two separate aircrafts over six months before authorization was approved for this installation. As can be seen in Fig. 13, optical fiber sensor allows safe and flexible routing around obstructions not possible with traditional sensors. Also, significant aircraft modification was not necessary, and the aircraft easily returned to the original configuration after the flights.

Fig. 14 shows the optical interface box and the data recorder system capable of up to 20 channels (with additional plugins). The systems were located inside the fuselage directly underneath where the fiber exited the cabin. Output from the optical box was filtered with a $2.5 \mathrm{MHz}$ low-pass filter for noise reduction before connected to two data recorder channels via a tee (splitter). One data recorder channel was configured for DC coupling. The second channel was configured for $\mathrm{AC}$ coupling (with $2 \mathrm{~Hz}$ internal high-pass filter) that was also used for system triggering. Triggering on the AC-coupled channel was more reliable at low current setting due to the slow output DC drift equivalent up to about 4000A observed during flight. The significant temperature swings at the box appeared to be the main cause of the DC drift. The effects on the measurement data would be minimal, however, as the drift amount can be easily subtracted from the data in post processing. Alternatively, the AC coupled data could be used instead but without the DC component of lightning. Builtin heater for temperature stabilization could be considered in future setups.

The system was flown on three flights that lasted 2.5 - 3 hours each. Occasionally the flight paths took the aircraft near thunderstorms; however, the aircraft was never struck by lightning. The system never suffered any false trigger, with the trigger level at the most sensitive setting of $1200 \mathrm{~A}$. This was expected since the system would only trigger when there was current flowing inside the fiber loop, i.e. on the fuselage. With proper fiber installation (closing the fiber loop), isolation from stray field would be excellent and false trigger was not a concern. 

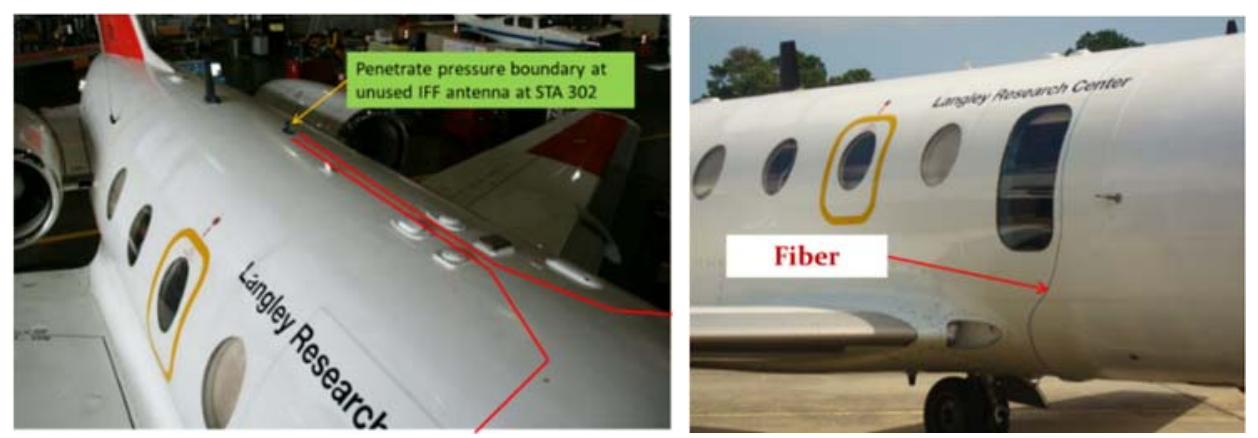

Figure 13. Fiber routing illustration atop of the aircraft. Fiber forms a loop encircling the aircraft fuselage forward of the aircraft wing (left). Final installation with fiber secured with polyurethane protective tape (right).
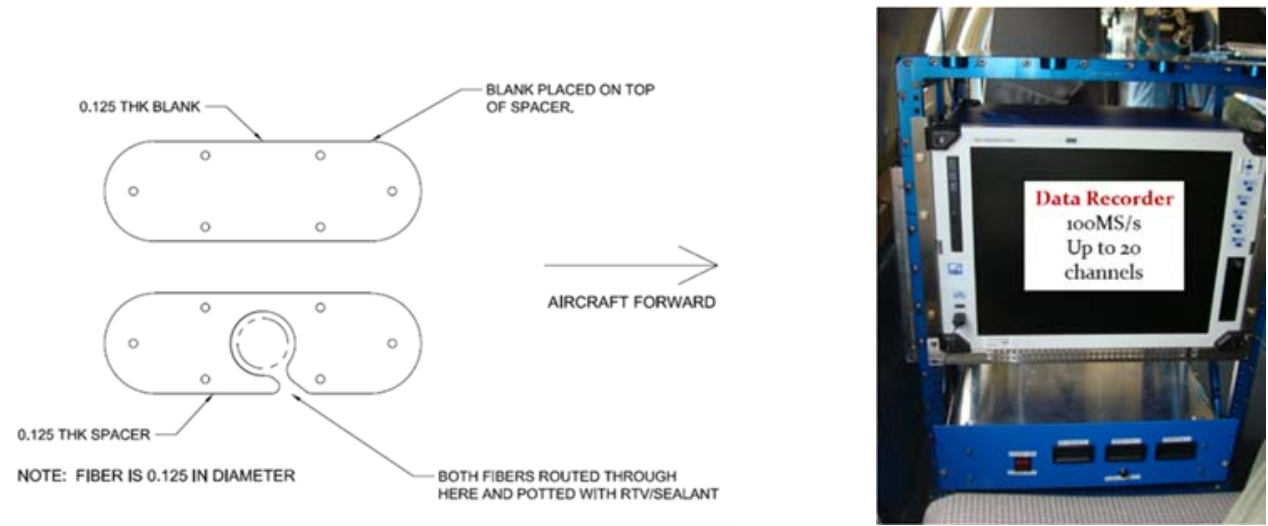

Figure 14. Aluminum spacer to allow fiber routing through an existing antenna port (left, bottom). Top plate can be a blank or an existing antenna. Data recorder and prototype optical interface box (right).

\section{CONCLUSION}

The design, accuracy, advantages and versatility of fiber optic current sensors based on Faraday Effect for lightning measurement were validated with three fiber-optic current sensor system operating at different wavelengths. Feasibility of aircraft installation and flights were demonstrated in three flights though the aircraft was not struck by lightning. These developments and demonstrations provide a better approach to characterize lightning parameters on aircraft and large structures. Many other lightning measurement applications are possible.

\section{REFERENCES}

[1] P. Laroche, P. Blanchet, A. Delannoy, F. Issac, "Experimental Studies of Lightning Strikes to Aircraft," Onera Aerospace Lab Journal, Issue 5, December 2012 (AL05-06).

[2] F. Pitts, B. Fisher, V. Mazur, and R. Perala, “Aircraft Jolts from Lightning Bolts,” IEEE Spectrum, July 1988.

[3] S. Alestra, I. Revel, V. Srithammavanh, M. Bardet, R. Zwemmer, D. Brown, N. Marchand, J. Ramos, V. Stelmashuk, V. "Developing an In-Flight Lightning Strike Damage Assessment System," Int. Conf. on Lightning and Static Electricity (ICOLSE), 2007.

[4] J. M. Lopex-Higuera, Editor. Handbook of Optical Fibre Sensing Technology, 2002; Sections 27.2 - 27.4.

[5] P. Drexler and P. Fiala,"Utilization of Faraday Mirror in Fiber Optic Current Sensors”, Radioengineering, Vol. 17, Dec. 2008.

[6] A. White, G. McHale, D. Goerz, "Advances in Optical Fiber-Based Faraday Rotation Diagnostics," 17th IEEE Int. Pulsed Power Conference, Wash. DC, July 2009 (LLNL-CONF-415198).

[7] R. Laming and D. Payne, "Electric Current Sensors Employing Spun Highly Birefringent Optical Fibers," Journal of Lightwave Technology, Dec. 1989.

[8] G.W. Day, M.N. Deeter and A.H. Rose, "Faraday effect sensors: A review of recent progress," Proc. SPIE 1779, 1992, pp. $21-26$.

[9] SAE ARP-5412 “Aircraft Lightning Environment and Related Test Waveforms," Rev. B, Jan 2012.

[10] V. Rakov, M. Uman, K. Rambo, “A Review of Ten Years of Triggered-Lightning Experiments at Camp Blanding, Florida," Atmospheric Research, Vol. 76, Issues 1-4, July-August 2005. 
[11] T. Nguyen, J. Ely, G. Szatkowski, C. Mata, A. Mata, and G. Snyder, "Fiber-Optic Current Sensor Validation with Triggered Lightning Measurements," 2013 Int. Conf. on Lightning and Static Electricity (ICOLSE).

[12] T. Nguyen, J. Ely, G. Szatkowski, C. Mata, A. Mata, G. Snyder, "An Intrinsic Fiber-Optic Sensor for Structure Lightning Current Measurement," 2014 Int. Lightning Detection Conference (ILDC).

[13] T. Nguyen, J. Ely, G. Szatkowski, C. Mata, A. Mata, G. Snyder, "Fiber-Optic Sensor for Aircraft Lightning Current Measurement,” 2012 Int. Conf. on Lightning Protection (ICLP). 